\title{
The influence of laparoscopic vs. open gastric bypass on hemodynamic function in morbidly obese patients during general anesthesia
}

\author{
Tomasz Gaszynski ${ }^{1}$, Tomasz Szewczyk ${ }^{2}$ \\ ${ }^{1}$ Department of Emergency and Disaster Medicine, Chair of Anesthesiology and Intensive Therapy, Medical University of Lodz, Poland \\ ${ }^{2}$ Department of Gastroenterological, Oncological and General Surgery, Barlicki University Hospital, Lodz, Poland
}

Videosurgery Miniinv 2014; 9 (1): 83-88 DOI: $10.5114 /$ wiitm.2014.40988

\begin{abstract}
Introduction: The open or laparoscopic procedure has an important influence on the hemodynamic function in morbidly obese patients undergoing bariatric surgery. The anesthesiologist and surgeon must be aware of changes in hemodynamic performance during laparoscopy or laparotomy under general anesthesia.

Aim: To evaluate and compare the hemodynamics in two types of surgery: open vs. laparoscopic.

Material and methods: After obtaining the local ethics committee approval 60 morbidly obese (MO) patients (body mass index $\geq 40 \mathrm{~kg} / \mathrm{m}^{2}$ ) scheduled for elective open or laparoscopic Roux-en-Y gastric bypass were included. Patients were allocated to study groups depending on the scheduled type of surgery (no randomization). General anesthesia with sevoflurane was performed. The hemodynamic parameters were recorded using a HemoSonic 100 device a transesophageal Doppler measurement at time points: T1 - initial, T2 - after creating pneumoperitoneum (group $P P$ ) or opening the abdomen (group $O P$ ).

Results: Complete data were collected on 28 patients in group LP and 21 in group OP. There was no statistical difference between groups in demographic data. At time point T2 in both groups the parameters stroke volume, peak velocity and cardiac index decreased, and total systemic vascular resistance increased significantly compared to $T 1$ $(p<0.05)$. In group LP the parameters cardiac output and acceleration (Acc) of blood decreased significantly compared to $T 1(p<0.05)$. There were significant differences between groups $(p>0.05)$ in cardiac output, total systemic vascular resistance and Acc, which were significantly higher in the OP group. In both groups mean arterial pressure increased and heart rate stayed similar to T1 with no significant difference ( $p>0.05)$. No complications were observed. Conclusions: Pneumoperitoneum has a significant negative influence on hemodynamic function during laparoscopic bariatric procedures compared to open surgery in morbidly obese patients.
\end{abstract}

Key words: obesity, anesthesia, laparoscopy, laparotomy, hemodynamics.

\section{Introduction}

If total body weight (TBW) corresponds with ideal body weight (IBW) the person is considered not to be obese. Ideal body weight may be defined as the body weight that provides the lowest health risk - risk of comorbidities leading to serious alteration in life important systems (cardiovascular, respiratory and metabolic) and as a result the lowest mortality for a given height, age, sex and frame size. The nutritional status of a person can be described by body mass index (BMI) [1].

People are considered obese when their BMI, a measurement obtained by dividing a person's weight in

\footnotetext{
Address for correspondence

Tomasz Gaszynski MD, PhD, Chair of Anesthesiology and Intensive Therapy, Barlicki University Hospital, 22 Kopcinskiego St,

90-153 Lodz, Poland, phone/fax: +48 4267837 48, e-mail: tomasz.gaszynski@umed.lodz.pl
} 
kilograms by the square of the person's height in meters, exceeds $30 \mathrm{~kg}$. A BMI of $\geq 40-49.9 \mathrm{~kg} / \mathrm{m}^{2}$ is morbid obesity, and a BMI of $\geq 50 \mathrm{~kg} / \mathrm{m}^{2}$ is super obesity.

A morbidly obese (MO) patient presents many unique challenges to the anesthetist. In addition to problems with venous access, patient positioning and airway control, obesity is associated with many cardiovascular conditions, which have important implications for the administration of anesthesia. There is an increase in the frequency of chronic diseases, such as systemic arterial hypertension, hypertensive heart disease, pulmonary arterial hypertension, and right and left ventricular failure. These conditions are very important during the perioperative period. Although there are some papers on the hemodynamic effect of pneumoperitoneum (PP) in the obese, those available do not compare the influence of different types of surgery - laparoscopic vs open - on cardiovascular function during general anesthesia for bariatric procedures.

\section{Aim}

The aim of the prospective observational study was to compare the influence of laparoscopic or open surgical procedures on hemodynamic function in MO during general volatile anesthesia.

\section{Material and methods}

Ethical approval for this study (RNN/257/03/KE) was provided by the Ethical Committee of Medical University of Lodz, Poland on 16 December 2003. Sixty patients scheduled for an elective laparoscopic or open Roux-En-Y gastric bypass procedure were included in the prospective study. Written informed consent from the study participants was obtained. Patients were divided into two groups based on

Table I. Demographic data

\begin{tabular}{|lcc|}
\hline Characteristics & Group LP & Group OP \\
\hline Gender (M/F) & $13 / 28$ & $7 / 14$ \\
\hline BSA [m²] & 2.5 & 2.42 \\
\hline Age [years] & 39.8 & 42.0 \\
\hline Weight [kg] & 134.0 & 138.3 \\
\hline Height [cm] & 169.2 & 167.3 \\
\hline BMI [kg/m²] & 45.9 & 46.2 \\
\hline Values are mean & & \\
\hline
\end{tabular}

scheduled surgery: laparoscopic (LP) or open (OP) no randomization was performed. Patients with coexisting cardiovascular diseases were excluded from the study, except for well-controlled hypertension induced by obesity. All patients were in ASA status 2 and NYHA 1 or 2.

Induction to anesthesia in both groups was performed with propofol $1.5-2.0 \mathrm{mg} / \mathrm{kg}$ of corrected body weight (CBW $=130 \%$ of IBW), fentanyl 100 $\mu \mathrm{g}$, midazolam $3 \mathrm{mg}$ and atracurium $0.6 \mathrm{mg} / \mathrm{kg}$ of IBW. Maintenance of anesthesia: All patients were anesthetized using sevoflurane. The patient's lungs were ventilated with a mixture of oxygen, air and sevoflurane in Vol\% depending on age and clinical parameters. In both groups repeated doses of fentanyl $0.005 \mathrm{mg} / \mathrm{kg}$ of CBW and atracurium $0.2 / \mathrm{kg}$ of IBW were administered intravenously.

Hemodynamic function was measured by a transesophageal Doppler probe using a HemoSonic 100 device (Arrow, USA). Measurements time points: T1 - after induction to anesthesia, T2 - insufflation of abdomen: creating PP (LP group, PP pressure was $15 \mathrm{~mm} \mathrm{Hg}$ ) or opening abdomen (OP group). Measured parameters: mean arterial pressure - MAP, heart rate - HR, acceleration - Acc, peak velocity PV, left ventricular ejection time - LVET, cardiac output - CO, stroke volume - SV; parameters adjusted for body surface area and age: total systemic vascular resistance - TSVR, cardiac index $-\mathrm{Cl}$. Measurements were taken 2-4 times at every time point and the mean was counted for analysis. Values of parameters at time points and differences between groups at time points were analyzed.

\section{Statistical analysis}

Statistical significance was set at $5 \%$. The distribution of data was tested with the Shapiro-Wilk test. Statistical analysis for parametric data was performed using the ANOVA test with Friedman modification, post-hoc Tukey test, and t-test, and for nonparametric data the Wilcoxon test. To compare changes in parametric data between groups the $t$-test was used and for nonparametric data the Mann-Whitney test.

\section{Results}

Complete data were collected in 28 patients in group LP and 21 in group OP. No complications were observed. There was no statistical difference between 
groups in demographic data (Table I). The results of measurements are presented in Table II. Initial hemodynamic parameters (T1) were similar in both groups with no significant difference $(p>0.05)$. At time point T2 in both groups the parameters SV, PV and $\mathrm{Cl}$ decreased, and TSVR increased significantly compared to T1 $(p<0.05)$. In group LP the parameters $\mathrm{CO}$ and Acc decreased significantly compared to T1 $(p<0.05)$. There were significant differences between groups $(p>0.05)$ in CO, TSVR and Acc, which were significantly higher in group OP. In both groups MAP increased and HR remained similar to $T 1$ with no significant difference $(p>0.05)$.

\section{Discussion}

The nutritional habits and status of the population are changing so morbid obesity is more and more common [1]. Obese patients have an abnormal cardiovascular status. Laparoscopy in bariatric surgery represents a modern method generally associated with lower morbidity and mortality, compared with the traditional surgical approach [2, 3]. However, in patients with impaired cardiovascular function, the laparoscopic approach is limited by the possible adverse hemodynamic impact. Although significant detrimental intra-operative hemodynamic and respiratory changes occur in MO during laparoscopic procedures there are no studies comparing the influence of laparoscopy and open surgery on hemodynamic function in MO patients.

The influence of PP on respiratory and cardiovascular systems was well described by Nguyen and Wolfe [4]. A morbidly obese patients have a 2 to 3 times higher intra-abdominal pressure than that of non-obese patients. Laparoscopic bariatric surgery requires abdominal insufflation with $\mathrm{CO}_{2}$ and an increase in the intra-abdominal pressure up to $15 \mathrm{~mm} \mathrm{Hg}$ with serious adverse consequences of PP. Laparoscopy in the MO, as in the non-obese, can lead to systemic absorption of $\mathrm{CO}_{2}$ and increased requirements for $\mathrm{CO}_{2}$ elimination. The increased intra-abdominal pressure enhances venous stasis, reduces intraoperative portal venous blood flow, decreases intraoperative urinary output, lowers respiratory compliance, increases airway pressure, and impairs cardiac function. Intraoperative management to minimize the adverse changes include optimizing anesthesia technique to minimize the effects of increased intra-abdominal pressure on renal and cardiac func-

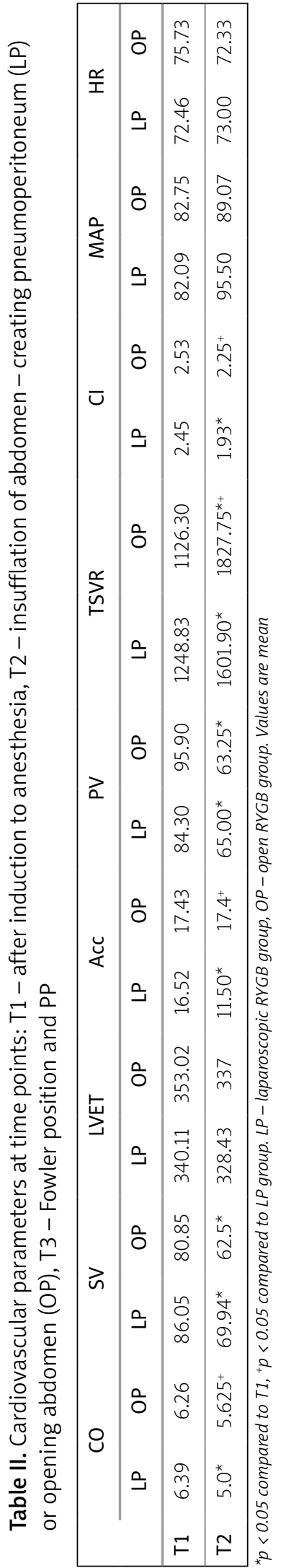


tion. A morbidly obese patients undergoing laparoscopic bariatric surgery are at risk for intraoperative complications related to the use of $\mathrm{CO}_{2}$ PP. However, many studies have demonstrated that obese patients who undergo laparoscopy have shorter hospital stays and fewer complications when compared with obese patients who undergo laparotomy [5]. With thorough preparation, careful preoperative evaluation and proper intraoperative anesthesia management, laparoscopy can be performed safely and is the preferred surgical method in MO. To the best of our knowledge this is the first study comparing intravenous and volatile anesthesia in respect of their influence on hemodynamic parameters during PP in MO.

Similarly to our findings, some authors observed significant hemodynamic and respiratory changes, mostly unfavorable, that occurred in the morbidly obese when creating the PP in preparation for laparoscopy in $\mathrm{MO}$ [6]. In other papers parameters such as CO increased - opposite to our results [7-9]. This increase in CO and MAP was associated with increase in HR probably induced by sympathetic stimulation. Interestingly, those authors obtained different results in the next published studies [10]. They observed a drop in $\mathrm{CO}$ and $\mathrm{Cl}$ after peritoneum $\mathrm{CO}_{2}$ insufflation, like we did. A drop in MAP, $\mathrm{HR}, \mathrm{Cl}$ and SVR during PP in MO was noted by Aloni et al., which was different to our results: an increase in MAP, HR and TSVR [11]. In their opinion the study confirmed the hypothesis that for most periods of laparoscopic surgery, obese patients are hemodynamically as stable as the non-obese. The authors point out that the anesthetic used may influence the hemodynamic parameters. In another study by Prior et al. left ventricular end systolic wall stress (LVESWS) was high during PP [12]. Since LVESWS is a determinant of myocardial oxygen demand, more aggressive control of blood pressure (ventricular afterload) in $\mathrm{MO}$ patients may be warranted to optimize the myocardial oxygen requirements. Fried et al. concluded that neither systolic nor diastolic performance was significantly affected by the introduction of capnoperitoneum and positioning of the MO patient for surgery [13]. They observed, opposite to our results, an increase in CO after creating PP. In a study published by Nguyen et al. CO was unchanged after insufflation (in our study $\mathrm{CO}$ decreased significantly after PP) [14]. In conclusion they claim that PP during laparoscopic gastric bypass does not impair cardiac function and is well tolerated by MO patients. Other authors came to the same conclusions [7, 11, 13]. Although some authors concluded that PP has significant effects on hemodynamics in $\mathrm{MO}$, they claim that these changes were of marginal clinical significance [8-10]. The hemodynamic changes are attenuated when the patient is placed in the reverse Trendelenburg position and almost completely corrected when the abdomen was deflated at the completion of the procedure $[6,12]$. When comparing the results of the above-mentioned papers we found some differences in methodology, anesthetics used and other important parameters including fluid load and PP pressure. In the majority of cited studies volatile anesthesia with isoflurane [7, 11] or sevoflurane [8-10] was performed. Only in one study was intravenous anesthesia with propofol performed [12]. The authors also used different methods of measurement of studied parameters: thermodilution with SwanGanz catheter [6, 7, 14], cardioimpedance [8-11] and transesophageal echocardiography $[12,13]$. There was no other study performed using a HemoSonic 100 device apart from ours. Those methods may be compared but there are some important differences. Bajorat et al. compared measurements taken with the HemoSonic 100 with PiCCO, NICO and SwanGanz catheters [15]. The results are similar, but HemoSonic 100 may show slightly lower values than thermodilution. The correlation was 0.84. Sawai et al. point out that arterial blood flow measured by HemoSonic 100 may change together with changes in $\mathrm{PaCO}_{2}$ which occurs during capnoperitoneum [16]. HemoSonic 100 transesophageal Doppler measurement is a non-invasive, easy to perform method. It is more reliable than older Doppler methods because it measures aorta diameter in real time. However, it has some disadvantages: it is very sensitive to the patient's movements. The axis between the echographic beam and aorta diameter should not exceed $20^{\circ}$. The recommended level of transducer is Th 6 but in some patients, such as the MO, it may be difficult to estimate it [17].

Fluid load in cited studies was similar and was between 5 and $10 \mathrm{ml} / \mathrm{kg}$ of IBW. In our study we administered $500 \mathrm{ml}$ of crystalloid before induction of anesthesia followed by infusion of $5 \mathrm{ml} / \mathrm{kg}$ of IBW. The PP pressure varied from $15 \mathrm{~mm} \mathrm{Hg}[11,14]$, $17 \mathrm{~mm} \mathrm{Hg}$ [4] up to $20 \mathrm{mmHg}$ [12]. In our study PP pressure was set at $15 \mathrm{~mm} \mathrm{Hg}$.

In the LP group the changes in cardiovascular parameters were similar to the results of other in- 
vestigators, who studied the influence of PP on hemodynamic function in $\mathrm{MO}$ anesthetized with one method, in most studies volatile anesthesia. In the OP group CO, PV and SV decreased significantly compared to T1. It may be due to placement of the Thompson retractor, which creates pressure on the diaphragm and mediastinum which may influence preload. However, the negative influence of this is not as significant as the influence of LP on hemodynamic parameters.

Our study confirms that PP has an important negative impact on hemodynamic function during general anesthesia in MO. After insufflation of abdomen contractility and flow parameters decrease significantly (CO, Acc, PV, SV), afterload parameters increase (TSVR). However, those changes are not clinically observed as serious cardiovascular disturbances. Blood pressure measured by standard means during laparoscopic procedures in $\mathrm{MO}$ does not provide precise information on cardiovascular hemodynamic function. Changes in contractility and flow parameters can only be estimated by advanced hemodynamic monitoring. Transesophageal Doppler monitoring is easy, safe and does not produce complications. It is precise enough to optimize anesthesia and fluid management during laparoscopic procedures in $\mathrm{MO}$, increasing safety of the patients.

The PP has an important negative impact on hemodynamic function during general anaesthesia in MO [18]. However, those changes are not clinically observed as serious cardiovascular disturbances. Open surgery creates less significant changes in important parameters such as $\mathrm{CO}$ and $\mathrm{Cl}$. There were important differences between studied groups regarding the influence on hemodynamic function during laparoscopy in MO patients or open surgery. This suggests that MO patients who suffer from cardiovascular diseases should be scheduled carefully for laparoscopic bariatric procedures. Open surgery gives not only more stable hemodynamic performance but also allows for lower pressures of mechanical ventilation during the bariatric procedure and general anesthesia in MO [19].

The anesthesiologist and surgeon, being aware of the influence of type of surgery on the cardiovascular system, should cooperate in the perioperative period: during cardiovascular preoperative evaluation and optimization, perioperative management of possible hemodynamic disturbances which may determine the success of the surgical procedure, and management of possible complications.

\section{Conclusions}

Pneumoperitoneum has a significant negative influence on hemodynamic function during laparoscopic bariatric procedures in morbidly obese patients compared to open surgery.

\section{References}

1. Jastrzębska-Mierzyńska M, Ostrowska L, Razak Hady H, Dadan J. Assessment of dietary habits, nutritional status and blood biochemical parameters in patients prepared for bariatric surgery: a preliminary study. Videosurgery Miniinv 2012; 7: 156-65.

2. Abalikšta T, Brimas G, Strupas K. Laparoscopic adjustable gastric banding. A prospective randomized study comparing the Swedish Adjustable Gastric Band and the MiniMizer Extra: oneyear results. Videosurgery Miniinv 2011; 6: 207-16.

3. Bobowicz M, Michalik M, Orłowski M, Frask A. Bariatric single incision laparoscopic surgery - review of initial experience. Videosurgery Miniinv 2011; 6: 48-52.

4. Nguyen NT, Wolfe BM. The physiologic effects of pneumoperitoneum in the morbidly obese. Ann Surg 2005; 241: 219-26.

5. Lamvu G, Zolnoun D, Boggess J, Steege JF. Obesity: physiologic changes and challenges during laparoscopy. Am J Obstet Gynecol 2004; 191: 669-74

6. Artuso D, Wayne M, Cassaro S, et al. Hemodynamic changes during laparoscopic gastric bypass procedures. Arch Surg (Chicago, Ill : 1960) 2005; 140: 289.

7. Dumont L, Mattys M, Mardirosoff C, et al. Hemodynamic changes during laparoscopic gastroplasty in morbidly obese patients. Obes Surg 1997; 7: 326-31.

8. El-Dawlatly AA, al-Dohayan A, Favretti F, Samarkandi A. Anaesthesia for morbidly obese patients: a study of haemodynamic changes during bariatric surgery. Middle East J Anesthesiol 2002; 16: 411-7.

9. El-Dawlatly A, Mansour E, Al-Shaer AA, et al. Impedance cardiography: noninvasive assessment of hemodynamics and thoracic fluid content during bariatric surgery. Obes Surg 2005; 15 : 655-8.

10. El-Dawlatly AA. Hemodynamic profile during laparoscopic cholecystectomy versus laparoscopic bariatric surgery: the impact of morbid obesity. Middle East J Anesthesiol 2006; 18: 733-42.

11. Aloni Y, Evron S, Ezri T, et al. Morbidly obese patients are hemodynamically stable during laparoscopic surgery: a thoracic bioimpedance study. J Clin Monit Compu 2006; 20: 261.

12. Prior DL, Sprung J, Thomas JD, et al. Echocardiographic and hemodynamic evaluation of cardiovascular performance during laparoscopy of morbidly obese patients. Obes Surg 2003; 13: 761-7.

13. Fried M, Krska Z, Danzig V. Does the laparoscopic approach significantly affect cardiac functions in laparoscopic surgery? Pilot study in non-obese and morbidly obese patients. Obes Surg 2001; 11: 293-6.

14. Nguyen NT, Ho HS, Fleming NW, et al. Cardiac function during laparoscopic vs open gastric bypass. Surg Endosc 2002; 16: 78-83.

15. Bajorat J, Hofmockel R, Vagts DA, et al. Comparison of invasive and less-invasive techniques of cardiac output measurement 
under different haemodynamic conditions in a pig model. Eur J Anaesthesiol 2006; 23: 23-30.

16. Sawai T, Nohmi T, Ohnishi Y, et al. Cardiac output measurement using the transesophageal Doppler method is less accurate than the thermodilution method when changing $\mathrm{PaCO} 2$. Anesth Analg 2005; 101: 1597-601.

17. Bernardin G, Tiger F, Fouche R, Mattei M. Continuous noninvasive measurement of aortic blood flow in critically ill patients with a new esophageal echo-Doppler system. J Crit Care 1998; 13: 177-83.

18. Gaszyński T. The effect of pneumoperitoneum on hemodynamic parameters in morbidly obese patients anaesthetized with Sevoflurane or Propofol: a randomized comparison study. Anest Inten Ther 2011; 43: 148-52.

19. Gaszyński T. The effects of abdominal opening on respiratory mechanics during general anaesthesia for open bariatric surgery in morbidly obese patients. Anest Inten Ther 2010; 42: 198-200.

Received: 6.10.2012, accepted: 13.08.2013. 